\title{
El cáncer en el cine. Algunas sugerencias más
}

\author{
J.E. Baños
}

He leído con atención el artículo de Icart-Isern et al [1] publicado recientemente en Educación Médica. Comparto con los autores la utilidad de las películas comerciales en mostrar aspectos de la práctica de la medicina que pueden ser difíciles de ilustrar y de comprender por los estudiantes de medicina u otras disciplinas sanitarias. En especial, es de agradecer el esfuerzo de los autores por recoger el máximo de películas sobre este tema y clasificarlas en función de la presencia más o menos importante de la enfermedad en su argumento. Obviamente, la obtención de una lista exhaustiva de todas ellas es una tarea difícil y quizás no del todo necesaria si se dispone de un número suficiente para ilustrar las situaciones que se desean mostrar. Sin embargo, me gustaría contribuir a aumentar las posibilidades de escoger mediante un número adicional de películas que se recogen en la tabla. Incluyen 16 más, la mayoría de origen anglosajón pero también algunas europeas, e incluso un par en castellano en su versión original. La mayoría se han obtenido de una base de datos de elevado interés que mantiene desde hace años la Facultad de Medicina de la Universidad de Nueva York, donde también se recogen obras literarias, de teatro o de otras actividades artísticas susceptibles de emplear en docencia [2]. Alguna de las otras películas, como La enfermedad de Sachs, se ha analizado en detalle por su utilidad para contribuir a explicar la relación médicoenfermo [3,4]. Aunque la utilidad y el interés de cada una depende de las circunstancias en que se utilicen, es difícil resistirse a aconsejar algunas de ellas. La única española, La princesa del polígono, tiene la ventaja añadida de contemplar las diferencias con la sociedad gitana en la consideración de la vida y de la enfermedad. Ikiru ('Vivir'), una pe- lícula antológica de Akira Kurosawa, es una obra maestra para reflejar la vivencia de la enfermedad en el paciente $[5,6]$ y está considerada como una de las mejores jamás rodada.

Queda el problema de su disponibilidad. En algunas ocasiones no se encuentran en versión española, ni tan solo subtituladas. En otras, son de imposible adquisición en España de forma directa; sin embargo, el acceso a Internet y las grandes posibilidades de un mundo globalizado permiten que conseguirlas sea mucho menos difícil que hace algunos años.

\section{Bibliografía}

1. Icart-Isern MT, Rozas-García MR, Sanfeliu-Cortés V, Viñas-Llebot H, Fernández-Ortega MP, Icart MC. El cáncer en el cine. Un recurso para los profesionales de la salud. Educ Med 2009; 12: 239-46.

2. Literature, Arts \& Medicine Database. Facultad de Medicina de la New York University. URL: http://medhum. med.nyu.edu. [09.01.2010].

3. Baños JE. How thick the shield should be: teaching the subtleties of the doctor-patient relationship using literature and popular movies. J Med Movies 2007; 3: 159-65.

4. Baños JE. How literature and popular movies can help in medical education: applications for teaching doctorpatient relationship. Med Educ 2007; 41: 918.

5. Young-Mason J. Revisiting Kurosawa’s Ikiru. Clin Nurse Spec 2004; 18: 51-2.

6. Yamada S, Maskarinec G, Greene G. Cross-cultural ethics and the moral development of physicians: lessons from Kurosawa's Ikiru. Fam Med 2003; 35: 167-9.
Facultad de Ciencias de la Salud y de la Vida. Universitat Pompeu Fabra. Barcelona, España.

Correspondencia Dr. Josep Eladi Baños Díez. Facultad de Ciencias de la Salud y de la Vida. Universitat Pompeu Fabra. Doctor Aiguader, 88. E-08003 Barcelona.

E-mail

josepeladi.banos@upf.edu 
Tabla. Películas en las que el cáncer se trata como tema principal o periférico.

\begin{tabular}{|c|c|}
\hline Título $^{a}$ & Director (año) \\
\hline The bucket list (Ahora o nunca) & Rob Reiner (2008) \\
\hline The belly of an architect (El vientre del arquitecto) & Peter Greenaway (1987) \\
\hline The green mile (La milla verde) & Frank Darabout (1999) \\
\hline A woman's tale (Historia de una mujer) & Paul Cox (1991) \\
\hline Ikiru (Vivir) & Akira Kurosawa (1952) \\
\hline Madame Curie & Melvin LeRoy (1943) \\
\hline Marvin's room (La habitación de Marvin) & Jerry Zaks (1996) \\
\hline La maladie du Dr. Sachs (La enfermedad de Sachs) & Michelle Deville (1999) \\
\hline Pieces of april & Peter Hedges (2003) \\
\hline La princesa del polígono & Rafael Montesinos (2007) \\
\hline Soft fruit & Christina Andreef (1999) \\
\hline La stanza del figlio (La habitación del hijo) & Nanni Moretti (2001) \\
\hline The vanishing line & Maren Grainger-Monsen (1998) \\
\hline Wilbur (Wilbur se quiere suicidar) & Lone Scherfig (2002) \\
\hline Y tu mamá también & Alfonso Cuarón (2001) \\
\hline The young doctors (Vivir es lo que importa) & Phil Karlson (1961) \\
\hline
\end{tabular}

